\title{
Body weight change and its association with food and beverage consumption during COVID-19 confinement: A study in 12 Ibero-American countries
}

\section{Brian M. Cavagnari}

Pontificia Universidad Católica Argentina

María Fernanda Vinueza-Veloz

Escuela Superior Politécnica de Chimborazo https://orcid.org/0000-0002-2493-0769

\section{Valeria Carpio-Arias}

Escuela Superior Politécnica de Chimborazo

Samuel Durán-Agüero

Universidad San Sebastián,

Isabel Ríos-Castillo

Organización de las Naciones Unidas para la Alimentación y la Agricultura

Edna J. Nava-González

Universidad del País Vasco

Patricio Pérez-Armijo

Saby Camacho-López

Universidad Privada Norbert Wiener

Jhon Jairo Bejarano-Roncancio

Universidad Nacional de Colombia

Beatríz Núñez-Martínez

Universidad Autónoma de Asunción

Gabriel González-Medina

Universidad de Las Américas

Sonia Ivankovich-Guillén

Universidad Católica del Uruguay

Alfonsina Ortíz

Universidad Católica del Uruguay

Karla Cordón-Arrivillaga

Universidad de San Carlos de Guatemala

\section{Eliana Romina Meza-Miranda}

Universidad Nacional de Asunción

Leslie Landaeta-Díaz ( $\sim$ llandaeta@udla.cl) 


\section{Research Article}

Keywords: COVID-19, confinement, food consumption, body weight, Ibero-America

Posted Date: May 21st, 2021

DOI: https://doi.org/10.21203/rs.3.rs-548724/v1

License: (c) (1) This work is licensed under a Creative Commons Attribution 4.0 International License. Read Full License 


\section{Abstract}

Background: SARS-CoV-2, a newly identified coronavirus responsible for the COVID-19 pandemic, has challenged health services and profoundly impacted people's lifestyle. The objective of the present study was to evaluate the effect of confinement during the COVID-19 pandemic on food consumption patterns and body weight in adults from 12 Ibero-American countries

Methods: Multicentric, cross-sectional study. Data was collected using an online survey disseminated by social networks. Sample included 10552 people from Spain and 11 Latin American countries who were selected by snowball sampling.

Results: While $38.50 \%$ of the sample reported weight gain, $16.90 \%$ reported weight lost. Weight change was associated to sex, age, country of residence and education level. People who were not confined, more often reported having maintained their weight in comparison to people who were confined. All Latin American countries showed an increased consumption of sweetened drinks, pastry products, fried foods and alcoholic beverages during confinement. Consumption of eggs and dairy products was independent from body weigh change. People who consumed more fruits and vegetables during the confinement more often reported having lose weight. In contrast, body weight gain during confinement was associated with increased intake of sugary drinks, baked goods and pastries, pizza, fried foods and alcoholic beverages.

Conclusions: During COVID-19 confinement all the Latin American countries included in this study showed a change in their consumption patterns toward less healthy diets, which in turn was associated with an increase in the body weight of their population.

\section{Introduction}

SARS-CoV-2 (severe acute respiratory syndrome coronavirus 2 ) is the virus that causes COVID-19 (coronavirus disease 2019)(1). This respiratory disease was declared a pandemic by the World Health Organization in March 2020 (2,3). Since then - but at different times, according to the epidemiological reality of each country - most nations adopted strict measures to prevent the spread of the infection and flatten the epidemic curve $(4,5)$. Such measures included border closures, social distancing, schools, squares and parks closures and voluntary or obligatory citizen's confinement (6).

These drastic measures lead people to stay at home more than usual, thus making physical activity difficult for both, children, and adults. Moreover, closure of stores made it difficult to acquire fresh food from nearby markets. Finally, the strong impact on family income specially on independent workers, due to the loss of employment led to the acquisition of poorer nutritional quality food products(7). While these measures were intended to avoid health problems inherent to the pandemic, they may have also caused a negative impact on other health aspects, both physical and psychological(7).

Moreover, and particularly concerning food and nutritional health, it has been described that prolonged confinement could lead to stress. Poor mental health may in turn modify food consumption patterns of 
the population, as well as their nutritional status, with the potential for individuals to gain or to lose weight. Although, the effect of confinement due to COVID-19 on eating habits and body weight in populations from different countries has been addressed, there is still controversy on this regard. While some studies show that during confinement dietary behaviors changed towards a healthier diet (8-10), others indicate trends towards less healthy patterns (10-13).

Given that we are facing an unprecedented situation in the recent world history and considering that many Latin American countries have a poor-quality basic diet from start (14), it is essential to understand how COVID-19 confinement may have influenced food consumption, diet quality and body weight in the region. Therefore, the objective of this study is to determine the effect of COVID-19 confinement on food consumption pattern and its association with body weight in an adult population of 12 lbero-American countries.

\section{Materials And Methods}

\section{Study design and setting}

The present work is a multi-center cross-sectional study. An online self-reporting survey with closed multiple-choice questions was used to collect data. Citizens from Spain and 11 Latin American countries (Argentina, Chile, Colombia, Costa Rica, Ecuador, Guatemala, Mexico, Peru, Paraguay, Panama, and Uruguay) were invited to participate in the study. Data collection took place between April 15th and May 4th, 2020. Each country had a health professional who coordinated data collection.

\section{Participants}

Participants over the age of 18 years old were included. Exclusion criteria included pregnant or nursing mothers, individuals in dietary or pharmacological treatment, individuals receiving psychotherapy for depression, anxiety disorders, stress, or mood disorders. Snowball sampling was used to recruit participants. In this way, invitations to participate in the study were sent through different platforms and social networks including, Facebook, Instagram, Twitter, emails and WhatsApp messages.

\section{Data source}

Data was collected using a self-administered survey via Google Forms. Participant responses are anonymous and confidential in accordance with Google's privacy policy(15). The survey was applied in Spanish and included questions regarding sociodemographic variables, body weight change, and food and beverage consumption frequency during COVID-19 confinement. To collect information on food and beverage consumption frequency, two previously validated questionnaires were used(16). A validation of the questionnaire was carried out by judgment of 25 experts, carried out through the Delphi method (17). To assess the validity of the questionnaire that was used in this study, the Cronbach's alpha was calculated, which was 0.77 . 
The English translated version of the survey can be found in Supplementary material 1.

\section{Variables}

Two main outcome variables were considered: body weight change and food consumption frequency. Answers regarding food consumption frequency from the survey were transformed into portions per week $(0,0.5,1,2,3,7,14,21)$. Food groups considered in the survey were sweetened beverages, dairy, fruits, vegetables, bread, baked goods, eggs, meat, coffee/tea, alcoholic beverages, fried foods and pizza(16). Body weight change was self-reported and evaluated by asking participants to select one of three options: increased, have not changed or decreased.

Main explanatory variables included: country of residence, age, sex, education level, residence area (urban or rural) and confinement length ( $<4$ weeks, $\geq 4$ weeks). We also analyzed the influence of human development by country using the Human Development Index (HDI) developed by the United Nations Development Program (UNDP) (18). According to this index, Spain, Argentina, Chile, Costa Rica, and Uruguay were included in the very high human development group; Colombia, Ecuador, Mexico, Panama, Paraguay, and Peru were included in the high human development group; and Guatemala in the medium human development group.

\section{Statistical analysis}

To investigate the factors associated with body weight change, a multinomial regression model was implemented. Body weight was included as a dependent variable. Country, sex, age, confinement length ( $<4$ weeks, $\geq 4$ weeks), education level (primary/secondary, university) and residence area (urban, rural) were considered as independent variables. In case of multiple comparisons, we established "Did not change" (body weight) and "Spain" (country) as reference levels. Spain was chosen as a reference for three reasons: First, Spain shares with Latin American countries language, costumes and traditions. Second, during COVID-19 pandemic, people from Spain have shown to adhere to healthier dietary patterns in comparison to people from other countries (9). Third, Spain shows the highest human development index (18).

To study the association between food consumption frequency and body weight, several regression models with restricted cubic splines (RCS) were implemented, with food consumption frequency as the dependent variable. One model was implemented for each of the food groups considered in the present study. Adjusted means of servings per week for each food group, with $95 \%$ confidence intervals $(95 \% \mathrm{Cl})$, were calculated. Other independent variables included were country, gender, age, confinement length, education level and residence area. All analyses were conducted using R-package and related packages, including rms $(19,20)$.

\section{Ethic declaration}


The study was developed following the Declaration of Helsinki to work with human beings and in accordance with the "Singapore Declaration on Integrity in Research". It was approved by the Scientific Ethics Committee of the University of the Americas (Chile) number 2020001 in a session on March 30th and reviewed by local committees in the participating countries. All participants accepted and provided an informed consent form included in the online form at the beginning of the survey.

\section{Results}

\section{Demographic characteristics of the population}

The study included 10552 individuals with a median age of 33 years, ranging from 18 to 86 , from whom $79.15 \%(n=8361)$ were women and $20.85 \%(n=2191)$ men. Most of participants were highly educated $(90.85 \%, n=9586)$, lived in urban areas $(91.64 \%, n=9656)$ and were totally confined when they answered the survey $(94.70 \%, n=9993)$. While $20.06 \%(n=2117)$ were confined for four weeks or less, $74.64 \%(n=$ 7876) were confined for more than four weeks. Most of individuals reported living in countries with high or very high $\mathrm{HDI}(47.22 \%(n=4983)$ and $43.66 \%(n=4607)$, respectively). Individuals who reported living in countries with medium human development corresponded to $9.12 \%(n=962)$. Demographic characteristics of the 12 countries included in the study are shown in Table 1.

\section{Characterization of body weight change}

More than half of the people reported having gain or lose weight $(55.40 \%, \mathrm{n}=5846)$. From the sample, $38.50 \%(n=4062)$ reported that their weight had increased and $16.91 \%(n=1784)$ that it had decreased. The percentage of males who reported maintaining or losing weight was higher than that of females (Did not change: $46.28 \%, n=1014$ vs. $44.16 \%, n=3692$; decreased: $18.30 \%, n=403$ vs. $16.54, n=1383$ ). In contrast, females more often reported having gained weight in comparison to males $(39.30 \%, n=3286$ vs. $35.42 \%, n=776$ ). Those who maintained their weight were older than those who lost weight but had a similar age than those who reported having gain weight (Did not change: 34 ; increased: 34 ; decreased: 31).

People with lower education level more often reported having maintained their body weight in comparison to people with a higher level of education $(46.27 \%, n=447$ vs. $44.43 \%, n=4259)$. In contrast, people with higher education level more often reported having increased or decreased weight than people with a lower level of education (Increased: $38.11, n=3652$ vs. $42.34 \%, n=409$; decreased: $17.46 \%, n=$ 1674 vs. $11.39 \%, n=110$ ). The percentage of people living in urban areas who reported maintaining, gaining, or losing body weight was similar than those of people living in rural areas (Did not change: $44.56 \%, n=4303$ vs $44.84 \%, n=395$; increased: $38.46, n=3714$ vs. $39.05 \%, n=344$; decreased: $16.96 \%$, $\mathrm{n}=1639$ vs. $16.12 \%, \mathrm{n}=142)$.

People who were not confined, more often reported having maintained their weight in comparison to people who were confined for $\leq 4$ weeks or for $>4$ weeks $(46.68 \%, n=261$ vs. $43.98 \%, n=931$ vs. $44.62 \%, n=3514$, respectively). Distribution of body weight increase was similar among people who were 
not confined, were confined for $\leq 4$ weeks or for $>4$ weeks $(39.18 \%, n=219$ vs. $39.21 \%, n=830$ vs. $39.26 \%, n=3013$, respectively). Countries with very high HDI, less often reported weight loss $(13.78 \%, n=$ $635)$ and more often reported weight gain $(41.20 \%, n=1898)$ than countries with high and medium HDI.

To investigate the association between self-reported body weight change and socio-demographic characteristics including country, sex, age, level of confinement, education level and residence area, we implemented a multinomial regression model (see Methods section). We observed that body weight change was significantly associated to country, sex, age, and education level, but not to confinement level or residence area (Table 2). In relation to the country of residence, and taking Spain as a reference, we found that living in Chile or Paraguay represented a greater risk of increasing body weight during COVID19 confinement: $81 \%$ and $88 \%$, respectively. Living in Colombia, on the other hand, represented a $22 \%$ lower risk. We also found that living in Ecuador and Panama represented a greater risk of losing weight during COVID-19 confinement: 57\% and 34\%, respectively. On the other hand, living in Argentina and Uruguay represented a lower risk: $27 \%$ and $59 \%$, respectively. Details of adjusted predicted probabilities of all countries can be seen in Supplementary Table 1.

Females as well as older people were at lower risk of increasing body weight in comparison to males or younger people (Table 2). Although the trend found in people with a higher education level was towards a lower risk of weight gain, differences were not statistically significant. In contrast, having a university education represented a $41 \%$ greater risk of weight loss during confinement.

\section{Food and beverage consumption patterns and their association with body weight change}

Consuming some foods (e.g., eggs and dairy products) during COVID-19 confinement was independent from body weight variations (Supplementary Table 2). People who consumed more fruits and vegetables during the confinement more often reported having lose weight. Conversely, body weight gain during confinement was associated with increased intake of sugared drinks, baked goods and pastries, pizza, fried foods, and alcoholic beverages (Figure 1). Consumption of sugared drinks, dairy and vegetables, but not the other food or beverages was associated with confinement level. People who were confined more than 4 weeks tended to consume less portions per week of sugared drinks than those who were confined less than 4 weeks or were not confined. The opposite was found in relation to dairy products and vegetables. People who were confined more than 4 weeks tended to consume more portions per week of dairy and vegetables than those who were confined less than 4 weeks or were not confined.

The percentage of the population of each country that reported having increased or decreased the consumption of certain types of foods and beverages is shown in figures $2 \mathrm{~A}$ and $2 \mathrm{~B}$, respectively. The same analysis, by food group, can be seen in the Supplementary Figures 1 (increased consumption) and 2 (decreased consumption).

It is interesting to note that those countries with very high human development (Argentina, Chile, Costa Rica, Spain, and Uruguay) doubled the intake frequency of vegetables and consumed half of the fried products, compared to the countries with high and medium human development, but also doubled the 
consumption of infusions, had a higher intake frequency of alcoholic beverages, and consumed a third of the legumes than the countries with medium human development. There were no significant differences in the consumption of other foods and beverages based on the human development index of each country (data not shown).

\section{Discussion}

Although results on the feeding behavior of different populations during the COVID-19 pandemic and its association with body weight variation during confinement have been recently reported $(3,6-13)$, according to our knowledge, these are the first data that analyze said changes in the Ibero-American population. In this study, we present the changes in the study population's body weight and food consumption patterns that have occurred during COVID-19 confinement, as well as their relation to various socio-demographic variables.

\section{Body weight variation during COVID-19 confinement}

Data from the United States of America indicate that $59 \%$ of the population reported having maintained their body weight, $22 \%$ reported having gained weight and $19 \%$ reported having lost weight (6). Our data are in line with those who presented a body weight decrease (16.9\%) but show a higher percentage of individuals who report having gained weight (38.5\%) and a lower percentage who report having maintained their body weight (44.6\%).

Several risk factors for weight gain during confinement have been described in the literature, such as eating snacks and sugary drinks after dinner, eating in response to stress, decreasing physical activity, significantly increasing television and screen time, and lack of sleep (6). It has also been described that stress can lead to cortisol release, which in turn increases the feeling of hunger (21). Other authors have pointed out that confinement interrupting the work routine could result in boredom, which has been associated with increased energy intake in general and fat and carbohydrate intake in particular (2). Finally, it has also been described that, during confinement, those individuals who gained weight may have eaten simply because food was available and not necessarily because of internal signs of hunger (6). In this regard, it is worth remembering that during the first weeks of the confinement and given the lack of knowledge of how long the pandemic could last, many cities showed the purchase of excess food to stockpile it, which may have led to an over-supply of food at home (3). Likewise, an increase in digital food trade through electronic home platforms has been reported (22).

Although in different reports most of the population refers to having gained weight, a percentage of the population losing weight can also be seen. In this sense, it also sounds plausible that many people stressed out by the confinement itself, by the fear of seeing the supermarket shortage or by the uncertainty of not knowing if they would continue with their employment or getting their full salary, may have channeled it through a decrease in food intake. Some countries such as the United States of 
America have reported that approximately $40 \%$ of the population decreased their food intake in response to stress(6).

Our results show no significant differences between body weight changes and confinement length. Perhaps, a longer follow-up could have shown a trend in this direction. At the time of preparation of this manuscript, most of the countries included in this study are still in confinement, so both weight gain and weight loss could probably affect a larger percentage of the population, which would be evident with longer follow-up.

As other authors point out (6), it would be important to analyze in future investigations, if the weight gained during confinement is maintained in time, once it is over. The same would apply to the weight lost during confinement.

\section{Consumption habits during COVID-19 confinement}

When associating the population weight gain or loss during confinement to the type and frequency of food consumed, it is not surprising that those people who lost weight consumed more fruit, while those who gained weight consumed more fried foods, baked goods, sweetened beverages, and alcoholic beverages.

People who gained weight also reported high consumption of infusions (mate, tea, coffee). In this context, in some Latin American countries, infusions are mostly consumed with the addition of table sugar. In fact, in countries such as Argentina, sugared infusions are the second source of added sugar, after sugary drinks (23). In line with the above, the literature describes how situations that have a strong impact on the population mood could lead people to overeat, looking mainly for sugary foods (2).

When comparing consumption habits during confinement, according to the human development index of the different participating countries, it is interesting to note that those countries with very high human development show a greater increase in the consumption of vegetables and a smaller increase in the consumption of fried foods. However, said countries also show the greatest increase in alcoholic beverages and infusions consumption.

Food consumption in countries with high and medium human development index does not differ significantly, except for legumes, whose consumption is higher in countries with medium level of human development. This could be explained by the food culture of these countries but also by the fact that these foods are more affordable and less perishable.

Some countries stand out for having increased the consumption of foods in a higher proportion than that reported on the rest of the countries included in this study. For example, Guatemala and Paraguay lead the percentage of the population that reports having increased consumption of sugar-sweetened beverages. The three countries with the highest percentage of their population reporting increased consumption of baked goods are Paraguay, Argentina, and Chile, with these last two countries also 
leading the increased chocolate consumption, which is probably justified since they are the southernmost countries in Latin America and that the survey was administered during winter.

\section{Consumption of alcoholic beverages during COVID-19 confinement}

The increase in the consumption of alcoholic beverages in the region deserves special analysis. Some authors raised two possible and opposite scenarios regarding the impact of the current COVID-19 pandemic on alcohol consumption (24). The first scenario predicts an increase in alcohol consumption in some populations, particularly men, due to psychological distress experienced because of the pandemic (financial difficulties, social isolation, and uncertainty about the post-pandemic future). The second scenario predicts a decrease in consumption based on less availability of alcoholic beverages - due to the closure of nearby stores - and on the reduced financial capacity to purchase them, due to reduced working hours and unemployment resulting from the pandemic crisis, which in turn would lead to a reduction in household income.

Our results indicate that Spain, Paraguay, Chile, Argentina, and Mexico are the countries that have increased beer consumption the most. Said five countries, along with Uruguay, are the ones reporting the greatest increase in alcoholic beverages consumption (wine and distillates), with Paraguay standing out with $7.8 \%$.

It is interesting to note that 4 out of these 6 countries have a very high human development index, so it is reasonable to think that the first proposed mechanism (increased consumption of alcoholic beverages) could predominate considering that the economic factor may not be the most relevant. Beyond this hypothesis, we must consider that, at the time of the study, the included countries were in different confinement phases and, since the proposed mechanisms are dynamic, one mechanism could prevail in a first phase and then become less relevant in more advanced confinement phases.

\section{Strengths and limitations}

The main limitation is that, due to strict guidelines of confinement and social distancing, the research had to be done through an online questionnaire, thus body weight changes being self-reported rather than being verified by a health professional. For the same reason, we do not have baseline BMI data, thus the clinical relevance of weight gains or loss at the individual level remains unknown.

Latin American countries do not have the same culinary traditions and eating behaviors, especially regarding their average daily consumption of legumes, nuts, fish, yogurt and sugary drinks $(14,23)$. Beyond these different starting points, the type and length of confinement has not been homogeneous throughout the region, thus being possible to find different eating behaviors between countries.

As the survey was sent through social networks, it could also be argued that the sample is not representative of the population, considering that many individuals may not have access to the internet. Finally, other limitations of the study have to do with the fact that the participants were mainly women and had a high education level. 
The great strength of this study lies in providing the first existing data at the Ibero-American level, regarding changes in the food consumption pattern during COVID-19 confinement and its association with the body weight of the population. As the present moment is unprecedented in history, beyond these results contributing to understanding the current situation, they also serve as a basis for designing public policies that can be used to prevent the potential negative health effects of confinement for future pandemics or other periods of global crisis.

\section{Conclusions}

During COVID-19 confinement, far from complying with eating a balanced and healthy diet, focused on fruits and vegetables, whole grains, vegetable and animal proteins, healthy fats and hydration based on safe water, the population of the 11 Latin-American countries that were included in this study showed a change in consumption patterns toward less healthy diets, increasing the consumption of sweetened drinks, pastry products, fried foods, and alcoholic beverages. In concordance, while $16.9 \%$ of the population reported losing weight, $38.5 \%$ reported a weight gain during COVID-19 confinement.

It would be in the interest of public health to carry out prospective studies to follow the population that has acquired these less healthy consumption patterns to see if, once the pandemic is over, these changes will end up generating new burdens of disease that, consequently, could put once again on test the Latin American health systems.

\section{Abbreviations}

AR: Argentina; CL: Chile; Co: Colombia; CR: Costa Rica; EC: Ecuador; GT: Guatemala; MX: Mexico; PA: Panama; PE: Peru; PY: Paraguay; SP: Spain; UY: Uruguay; Alc: alcoholic beverages; Bak: bakery; Choc: chocolate; DSD: diet soft drinks; FF: fried foods; Fru: fruits; Leg: legumes; PF: fried potatoes; Piz: pizza, SD: soft drinks, Yog: yogurt; Coo: Cookies; Dai: dairies; Fis: Fish; Fru: fruits; Mea: meat; Swe: Sweets; Veg: vegetables; QR: Interquartile Range, n: population size; OR: Odds Ratio

\section{Declarations}

\section{Ethical Standards Disclosure}

This study was conducted according to the guidelines laid down in the Declaration of Helsinki and all procedures involving research study participants were approved by the Scientific Ethics Committee of the University of the Americas (Chile) number 2020001 in a session on March 30th and reviewed by local committees in the participating countries. All participants accepted an informed consent form included in the online form at the beginning of the survey.

\section{Consent for publication}

Not applicable. 
The datasets used and/or analyzed during the current study are available from the corresponding author on reasonable request.

Conflicts of Interest: BMC has received fees, consulting payments, and unrestricted research funding from government and non-profit sources. He has also received consulting fees from biotechnology, pharmaceutical, and food and beverage companies. None of the above entities had any role in the design or preparation of this manuscript. The remaining authors have no conflict of interest to declare.

Financial Support: This research received no external funding.

Author Contributions: B.C., V.C-A., MF.Z. and S.D; Conceptualization. Methodology, formal analysis, investigation, data curation, writing, original draft, writing, review and editing. L. L-D: Conceptualization. Methodology, formal analysis, investigation, data curation, writing, original draft, writing, review and editing and Project administration. I.R-C., E. N-G., P.P-A., S. C-L, S.M-A., J.B-R., B. N-M., G. G-M., S.I., A.O., K. C-A., E. M-M., L. L-D: Methodology, Investigation, Writing - Review \& Editing.

Acknowledgments: To Jorge Morales for his collaboration in the making of the figures.

\section{References}

1. Butler MJ, Barrientos RM. The impact of nutrition on COVID-19 susceptibility and long-term consequences. Brain Behav Immun. 2020;(January).

2. Muscogiuri G, Barrea L, Savastano S, Colao A. Nutritional recommendations for CoVID-19 quarantine. Eur J Clin Nutr [Internet]. 2020;74(6):850-1. Available from: http://dx.doi.org/10.1038/s41430-020-0635-2

3. Abbas AM, Kamel MM. Dietary habits in adults during quarantine in the context of COVID-19 pandemic. Obes Med. 2020;(January):19-21.

4. Viner RM, Russell SJ, Croker H, Packer J, Ward J, Stansfield C, et al. School closure and management practices during coronavirus outbreaks including COVID-19: a rapid systematic review. Lancet Child Adolesc Heal [Internet]. 2020;4(5):397-404. Available from: http://dx.doi.org/10.1016/S23524642(20)30095-X

5. WHO. Rational use of personal protective equipment for coronavirus disease 2019 (COVID-19) and considerations during severe shortages. Who [Internet]. 2020;(April):1-28. Available from: https://apps.who.int/iris/handle/10665/331695

6. Zeigler Z, Forbes B, Lopez B, Pedersen G, Welty J, Deyo A, et al. Self-quarantine and weight gain related risk factors during the COVID-19 pandemic. Obes Res Clin Pract. 2020;14(May-June):210-6.

7. Martinez-Ferran M, de la Guía-Galipienso F, Sanchis-Gomar F, Pareja-Galeano H. Metabolic impacts of confinement during the COVID-19 pandemic due to modified diet and physical activity habits. Nutrients. 2020;12(6). 
8. Ruiz-Roso MB, Padilha P de C, Mantilla-Escalante DC, Ulloa N, Brun P, Acevedo-Correa D, et al. Confinamiento del Covid-19 y cambios en las tendencias alimentarias de los adolescentes en Italia, España, Chile, Colombia y Brasil. Nutrients. 2020;12(6):1-18.

9. Rodríguez-Pérez C, Molina-Montes E, Verardo V, Artacho R, García-Villanova B, Guerra-Hernández EJ, et al. Changes in dietary behaviours during the COVID-19 outbreak confinement in the Spanish COVIDiet study. Nutrients. 2020;12(6):1-19.

10. Sánchez-Sánchez E, Ramírez-Vargas G, Avellaneda-López Y, Orellana-Pecino Jl, García-Marín E, DíazJimenez J. Eating habits and physical activity of the spanish population during the covid-19 pandemic period. Nutrients. 2020;12(9):1-12.

11. Scarmozzino F, Visioli F. Covid-19 and the subsequent lockdown modified dietary habits of almost half the population in an Italian sample. Foods. 2020;9(5).

12. Villaseñor, K. Jimenez, A, Ortega, A. Islas, L. Gonzales O. Silva T. Cambios en el estilo de vida y nutrición durante el confinamiento por SARS-CoV- 2 ( COVID-19) en México: Un estudio observacional. RevEsp Nutr Hum Diet [Internet]. 2020;25:1-21. Available from: http://ww.renhyd.org/index.php/renhyd/article/view/1099/662

13. Reyes-Olavarría D, Latorre-Román PÁ, Guzmán-Guzmán IP, Jerez-Mayorga D, Caamaño-Navarrete F, Delgado-Floody P. Positive and negative changes in food habits, physical activity patterns, and weight status during covid-19 confinement: Associated factors in the chilean population. Int $\mathrm{J}$ Environ Res Public Health. 2020;17(15):1-14.

14. Kovalskys I, Zonis L, Guajardo V, Rigotti A, Koletzko B, Fisberg M, et al. Latin American consumption of major food groups: Results from the ELANS study. PLoS One. 2019;14(12).

15. Privacy Policy - Privacy \& Terms - Google.

16. Durán Agüero S, Valdés B. P, Godoy C. A, Herrera V. T, Herrera V. T. Hábitos alimentarios y condición física en estudiantes de pedagogía en educación física. Rev Chil Nutr. 2014;41(3):251-9.

17. Linstone HA TM. The Delphi method: Techniques and applications. Reading. MA Addison WEsley Publ. 1975;

18. Programa de las Naciones Unidas para el Desarrollo. Informe sobre Desarrollo Humano 2019. International Affairs. Nueva York, NY 10017, Estados Unidos; 2019.

19. Harrell Jr FE. rms: Regression Modeling Strategies [Internet]. [cited 2020 Sep 23]. Available from: https://cran.r-project.org/package=rms

20. CoreTeam R. R: A Language and envionment for statical computing. [Computer software]. Vienna, Austria; 2018.

21. Gluck ME, Geliebter A, Hung J, Yahav E. Cortisol, hunger, and desire to binge eat following a cold stress test in obese women with binge eating disorder. Psychosom Med. 2004;66(6):876-81.

22. Hobbs JE. Food supply chains during the COVID-19 pandemic. Can J Agric Econ. 2020;68(2):171-6.

23. Kovalskys I, Cavagnari BM, Favieri A, Guajardo V, Gerardi A, Previdelli ÁN, et al. Main sources of added sugars in Argentina. Medicina (B Aires). 2019;79(5):358-66. 
24. Rehm J, Kilian C, Ferreira-Borges C, Jernigan D, Monteiro M, Parry CDH, et al. Alcohol use in times of the COVID 19: Implications for monitoring and policy. Drug Alcohol Rev. 2020;39(4):301-4.

Figures

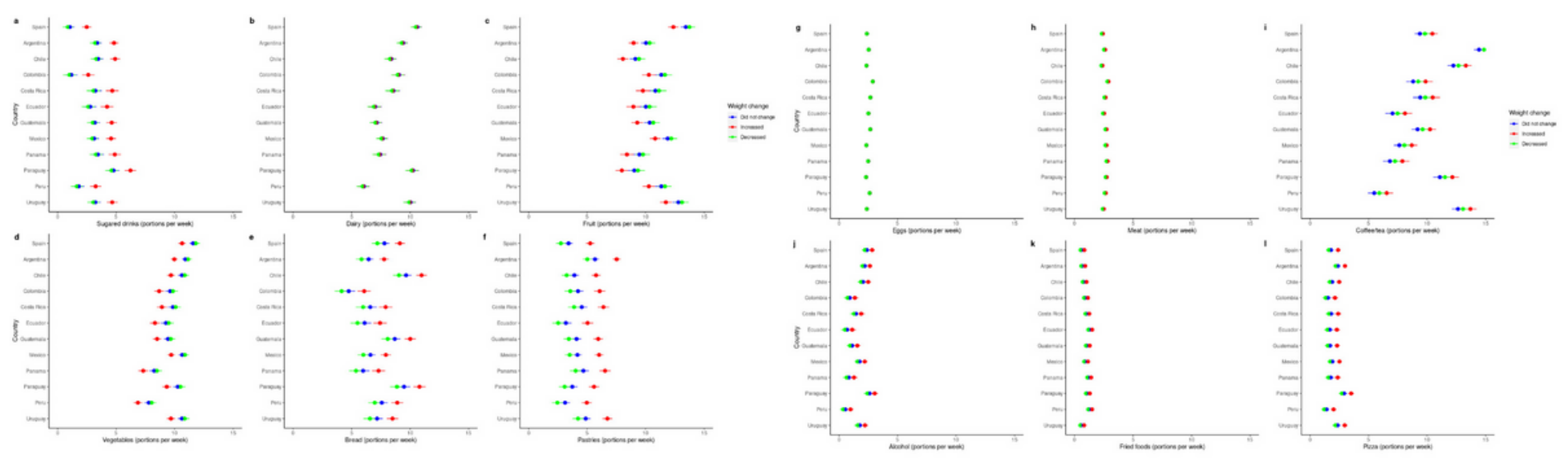

Figure 1

Food frequency consumption by country during COVID-19 confinement Footnote: Adjusted mean frequencies were calculated based on coefficients of RCS's models (see Methods section). Models were adjusted by sex, age, confinement length, education level, and residence area.

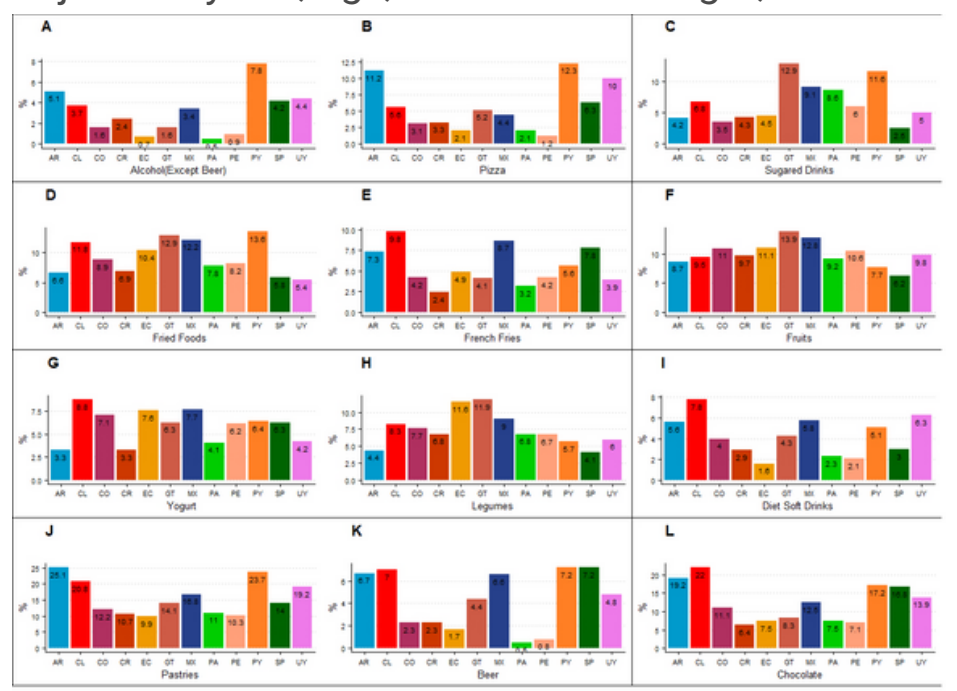

a

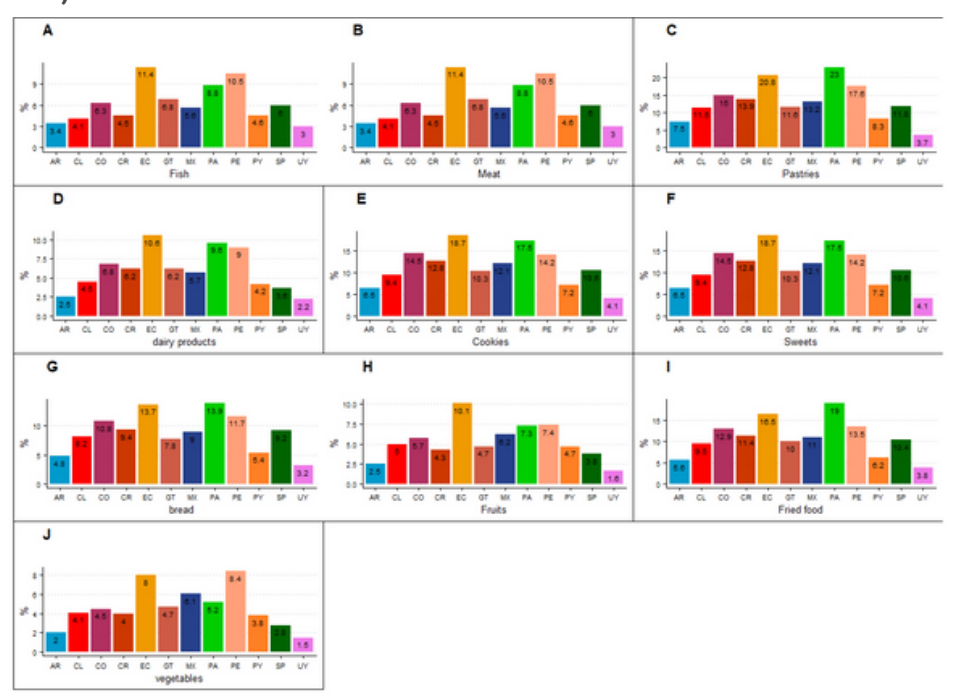

b

Figure 2

A: Percentage of the population of each country that reported having increased the consumption of different foods and beverages during COVID-19 confinement Footnote: AR: Argentina; CL: Chile; CO: Colombia; CR: Costa Rica; EC: Ecuador; GT: Guatemala; MX: Mexico; PA: Panama; PE: Peru; PY: Paraguay; SP: Spain; UY: Uruguay B: Percentage of the population of each country that reported having decreased the consumption of different foods and beverages during COVID-19 confinement Footnote: AR: Argentina; 
CL: Chile; CO: Colombia; CR: Costa Rica; EC: Ecuador; GT: Guatemala; MX: Mexico; PA: Panama; PE: Peru; PY: Paraguay; SP: Spain; UY: Uruguay

\section{Supplementary Files}

This is a list of supplementary files associated with this preprint. Click to download.

- SupplementaryFigure1.jpg

- SupplementaryFigure2.jpg 